\title{
Hypothesis on Pathophysiologic Mechanisms in COVID-19 Rare Ophthalmic Presentations; A Review
}

\author{
Sasan Jafari ${ }^{1}$, Masood Bagheri ${ }^{2 *}$ and Ahad Jafari ${ }^{1}$ \\ ${ }^{1}$ Department of Ophthalmology, Nikookari Eye Center, Tabriz University of Medical \\ Sciences, Tabriz, Iran \\ ${ }^{2}$ Department of Ophthalmology, Imam Khomeini Eye Center, Kermanshah \\ University of Medical Sciences, Kermanshah, Iran \\ *Corresponding Author: Masood Bagheri, Department of Ophthalmology, Imam \\ Khomeini Eye Center, Kermanshah University of Medical Sciences, Kermanshah, \\ Iran.
}

Received: March 18, 2021

Published: April 25, 2021

(C) All rights are reserved by Masood

Bagheri., et al.

\section{Abstract}

In late 2019, the world encountered the novel coronavirus severe acute respiratory syndrome (COVID-19) pandemic. Ophthalmic findings in COVID-19 patients were mainly ocular surface initially. Over time, different ocular involvements were reported in this pandemic. The aim of this study was to review the variety of possible ocular involvements with underlying pathophysiologic hypotheses in COVID-19. This study demonstrates the variety of suspicious ocular involvements in COVID-19 from ocular irritation to visual loss in three different categories: direct infectious ocular involvement, indirect ophthalmic complications and therapeuticprophylactic options ocular side effects.

This review represents a panoramic spectra of COVID-19 ophthalmic symptomatology with possible underlying pathophysiology.

Keywords: Coronavirus; 2019-nCoV; COVID19; SARS-CoV-2; Epidemic; Pandemic; Ophthalmology

\section{Introduction}

On $30^{\text {th }}$-January, 2020, the WHO declared an epidemic of new emerging pneumonia outbreak, that was named COVID-19 (2019nCoV or SARS-CoV-2), as a Public Health Emergency of International Concern (PHEIC), and in 11 $1^{\text {st }}$-March, 2020, announced as a pandemic [1]. According to the latest data available (on $4^{\text {th }}$-February, 2021), the cumulative number of confirmed infected cases are $104,745,839$ across 219 countries with 2,272,691 deaths.

In recent pandemic most of patients have mild symptoms with good prognosis, so far, a few patients progress to severe illness and even death. Patients infected with COVID-19 commonly develop fever, cough, myalgia, fatigue, and dyspnea; However, the range of clinical presentations is much wider [2].

The COVID-19 transmitting route is mainly respiratory by droplet with high infectivity, similar to the other two zoonotic epidemics by corona viruses ( $\mathrm{CoV})$, severe acute respiratory syndrome (SARS) and Middle East respiratory syndrome (MERS) [3]. Other 3 highly suspected ways to transmit are discussed as: aerosol, environmental surfaces and Fecal-oral or fecal-droplet [4]. Patientphysician close contact during ophthalmic examination may put them at higher risk of COVID-19 transmission [5]; this is while we know respiratory droplets followed by coughing and sneezing can 
be spread for up to $6 \mathrm{~m}$, a range that definitely exceeds the distance between the patient-ophthalmologist [6]. On the other hand, mostly ophthalmic centers are extremely crowded and commonly patients have to stay for a long time for a whole examination that may involve multiple investigations such as visual acuity measurement, fundus exam with pupillary dilatation and paraclinic imaging. All these factors increase the likelihood of cross-infection, between patients and patients-health workers. Therefore, recognizing the different manifestations of the disease is important not only in the timely diagnosis and treatment of patients but also for preventive measures.

According to the emerging evidence suggesting conjunctivitis as one of the early symptoms of COVID-19 on the one hand and anecdotal recent reports declared detection of responsible coronavirus RNA on tear samples from affected patients on the other hand, raised the likelihood of ocular involvement during this infection.

In view of the emerging evidence surrounding COVID-19 ophthalmologic presentation we performed this brief review to highlight the various ocular manifestations have been reported, which can help in the correct diagnosis of patients and taking preventive measures to minimize COVID-19 cross-infection between patients and patient-healthcare.

\section{Materials and Methods}

We conducted a literature search in the MEDLINE, EMBASE and Google Scholar up to January 2021. Keywords “COVID-19” or "2019 novel coronavirus" or "SARS-CoV-2" in combination with "eye”, "ophthalmology”, "ophthalmic", "ocular," and "orbital” were used. All reports about the ocular manifestations of patients with COVID-19 were enrolled. We did a main point summary with the title, author and the most important points of each study.

\section{Results}

With the progress of this pandemic the clinical spectrum of COVID-19 presentation continues to evolve. Ophthalmic findings in COVID-19 were mainly ocular surface initially [7,8]; however, with the passage of time, different ocular involvement was reported in this pandemic. Ocular manifestation in COVID-19 patients can be classified into three categories: direct infectious involvement, indirect disease complications, and ocular side effects of prescribed therapeutic-prophylactic options (Figure 1 and 2).

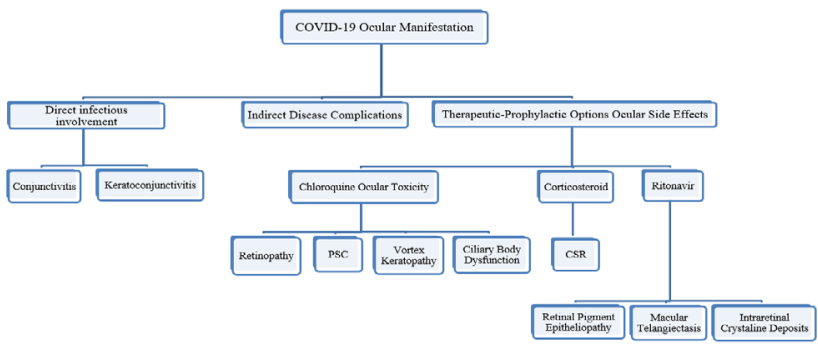

Figure 1: In this figure ocular manifestation reported in COVID-19 patients classified into three categories. Subset of direct infectious ocular involvements and therapeutic -prophylactic ocular side effects showed in this diagram. (PSC:

Posterior Subcapsular Cataract, CSR: Central Serous Chorioretinopathy).

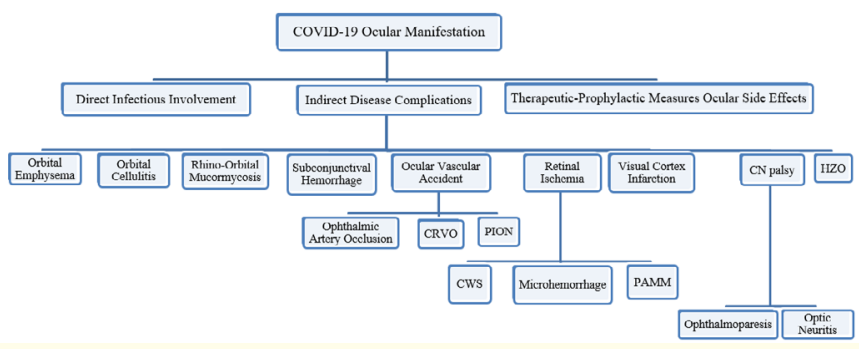

Figure 2: In this figure ocular manifestation reported in COVID-19 patients classified into three categories. Subset of indirect disease complications showed in this diagram. (CN palsy: Cranial Nerve Palsy, HZO: Herpes Zoster Ophthalmicus, CRVO: Central Retinal Vein Occlusion, PION: Posterior Ischemic Optic Neuropathy, CWS: Cotton Wool Spot, PAMM: Paracentral Acute Middle Maculopathy).

\section{Direct infectious involvement}

In 2003, CoV RNA showed in tear samples in SARS patients in Singapore [9]. Similar concerns have been mentioned with COVID-19. Actually, COVID-19 have been detected in conjunctival samples of both patients with and without conjunctivitis; even mild 
conjunctivitis can be the first and only symptom of the COVID-19 [10]. Gradually, several studies reported other ocular symptoms include: dry eye, foreign body sensation, "ophthalmalgia", etc. as direct manifestations of infection [7,11,12].

\section{Conjunctivitis}

The COVID-19 can infect the conjunctival epithelium through the droplets and body fluids [13]. Conjunctivitis has been reported as a prevalent well known ocular symptom in patients with $\mathrm{CO}$ VID-19 in several studies [7,11,12,14-16] and estimated a third of patients had ocular abnormalities, which frequently occurred in more severe cases [7]. Even mild conjunctivitis can be the first and only symptom [10]. The hypothesis, as in other viral cases, is a direct invasion of the conjunctival tissue which causes a follicular conjunctivitis.

\section{Keratoconjunctivitis}

A study by Cheema., et al. [17] has demonstrated keratoconjunctivitis as the main presentation of COVID-19 in a 29 years old female. The patient presented with the follicular conjunctivitis and diffuse corneal epithelial defects and subepithelial infiltrates. Bacterial cultures were negative. Besides previous study, Guo., et al. [18] demonstrated a COVID-19 patient with a relapsing keratoconjunctivitis. At first, the patient had only a left conjunctivitis about 10 days after the onset of SARS-CoV-2 infection.

The purpose of this study is to investigate possible different ocular conflicts in COVID-19, so ocular surface diseases which have known causal relationship mentioned briefly.

\section{Indirect disease complications}

COVID-19 may increase the risk of systemic diseases like diabetes mellitus (DM) and Kawasaki disease which is followed by the development or exacerbation of ocular symptoms. Also, ocular complications can occur for intensive care units like ocular surface disorders, secondary infections, acute ischemic optic neuropathy and raised intraocular pressure.

\section{Ocular vascular accident}

Ocular vascular accident in COVID-19 patients firstly reported by Oana M Dumitrascu and colleagues [19] in a 48 years old man who suffered from deep venous thrombosis (DVT) followed by ophthalmic artery occlusion. Another study by Gaba WH., et al. reported bilateral central retinal vein occlusion (CRVO) in a 40 years old man, known case of COVID-19 [20]. Also, Walinjkar JA., et al. presented a 17 years old female with unilateral CRVO in the absence of any known etiological factors other than recently COVID-19 [21]. Another study by Selvaraj V and colleagues reported a newly diagnosed COVID-19 female with acute, painless unilateral visual disturbance [22]. Given her ophthalmic exam they considered, posterior ischemic optic neuropathy (PION) was more likely. Three different types of PION have been described including: nonarteritic, arteritic and surgical. The capillary plexuses supplying the posterior part of the optic nerve are vulnerable to hypoperfusion and ischemia, so hypotension in critical COVID-19 patients, complement mediated microvasculopathies or purely hypercoagulability state hypothesized as probable mechanisms in ischemic optic neuropathy events.

The significant changes at the level of the retina have also been reported in COVID-19. They include hyperreflective lesions in the inner layers on optical coherence tomography (OCT), cotton-wool spots, and microhemorrhages as well as paracentral acute middle maculopathy (PAMM). The PAMM is an ischemia of the deep retinal circulation with hyperreflective band-like lesions at inner nuclear layer and outer-plexiform layer on spectral domain OCT (SD-OCT) $[23,24]$.

This infectious complication can be catastrophic, leading to both arterial and venous occlusion, microinfarcts, and even multiorgan failure. The higher incidence of thrombotic complications in these patients was related to multiple predisposing factors which also associated with poor prognosis $[25,26]$. Although the pathogenesis is not entirely understood, the presumed mechanisms were thrombophilia, vascular endothelial dysfunction and cardioembolic etiology (due to cardiomyopathy or arrhythmia) associated with the acute inflammatory response in COVID-19 patients. The hypercoagulable state in COVID-19 may be unique and distinct from other virus-related or sepsis-induced thrombophilia and considerable investigation recommended. Evidence of vascular thromboembolic accidents in COVID-19 recommended the prophylactic anticoagulation for all admitted patients [27]. Even a systematic review on severe COVID-19 patients recommended therapeutic anticoagulation for prevention of VTE and declared prophylactic dose is insufficient [26]. The proper management algorithm for the COVID-19-associated coagulopathies and the best regimen for thromboembolic events prevention is controversial. 


\section{Cerebrovascular accident (CVA)}

Vascular accidents accompanied with COVID-19 are not limited to the eye, but other vascular disorders like CVAs may influence the vision. Cyr., et al. reported two COVID-19 patients with such complication [28]. These patients experienced sudden vision loss due to acute occipital visual cortex ischemia or central visual pathway involvement.

As ophthalmologists, we encounter another probable destructive comorbidity in COVID-19 infection with a serious visual threatening consequence. The COVID-19 associated hypercoagulability state can induce thromboembolic events with significant visual deficits, which may cause debilitating sight-loss. As ophthalmologist it is critical to be wary about these rare neuro-ophthalmic comorbidities, and act in an integrated multidisciplinary approach for proper early diagnosis with appropriate management. The role of anticoagulants in the management of these complications requires subsequent extensive clinical studies.

\section{Cranial nerve (CN) palsy}

According to reported cases with COVID-19 infection, CN involvement is also possible. Belghmaidi., et al. [29] showed $3^{\text {rd }}$ nerve palsy in a previously healthy 24 years old female. Another study by Dinkin., et al. [30] reported two patients with COVID-19 presented with cranial neuropathies. First one was a 36 years old male with a unilateral partial $3^{\text {rd }}$ nerve palsy and bilateral $6^{\text {th }}$ nerve palsy. The second one was a 71 years old female with unilateral $6^{\text {th }}$ nerve palsy. Also isolated $6^{\text {th }} \mathrm{CN}$ palsy described by Greer and colleagues in two patients as COVID-19 possible comorbidity without any other risk factors [31].

Benito., et al. reported a case of optic neuritis as a comorbidity in a COVID-19 patient [32]. One of the known differential diagnosis of the optic neuropathy with anterior optic disc edema is viral optic neuritis specially in younger age. Like other viral agents, COVID-19 can act as a predisposing factor for optic neuritis, however, this report shows the involvement of a middle-aged patient.

\section{Orbital emphysema}

Shah., et al. and Stevens DV., et al. in two separated reports present spontaneous non-traumatic orbital emphysema in patients following COVID-19 infection [33,34]. A 42 and a 61 years old men, were also developed orbital emphysema following mechanical ventilation due to COVID-19 pneumonia induced acute respiratory distress syndrome (ARDS) [35,36].

Forceful blowing which increases the intranasal pressure, accompanied by age related bony changes in lamina papyracea or barotrauma in a ventilated patient with COVID-19 pneumonia can be the explanations for this complication. Refractory hypoxia in severely pneumonia prompted increased levels of positive end expiratory pressure (PEEP) in intubated patients are hypothesized as predisposing factors for orbital emphysema. The importance of orbital emphysema is about orbital compartment syndrome or orbital cellulitis due to superinfection, so patients should be avoided any valsalva maneuver and prophylactic antibiotic prescribe $[37,38]$.

\section{Orbital cellulitis}

In a study by Turbin., et al. [11] orbital cellulitis has been shown in two patients with COVID-19. A 12 and a 15 years old males diagnosed with COVID-19 showed progressive painful unilateral orbital edema. None of them had the symptoms of chronic sinusitis, but orbital computed tomography (CT) scan and magnetic resonance imaging (MRI) showed the acute invasive paranasal sinusitis.

Prolonged mucociliary clearance in patients with COVID-19 has reported [39]. We hypothesize that secondary upper respiratory tract congestion due to COVID-19 has been responsible for secondary sinus obstruction and bacterial orbital cellulitis in these patients. CoV infectious damage to the respiratory epithelium and ciliary dyskinesia may occur. Also prolonged mucociliary transport along with immunocompromised states and mechanical obstruction promotes bacterial overgrowth and predisposes patients to superinfection, bacterial sinusitis and orbital cellulitis. Low oxygen tension within an obstructed sinus cavity exacerbates this process leading to preseptal or orbital inflammation. Complexity of the mentioned mechanisms can be considered for the cases in Turbin's study.

\section{Rhino-orbital mucormycosis}

Mehta., et al. [40] reported a 60 years old male with a long-term diabetes mellitus (DM) and positive COVID-19 infection which progressd to bilateral lid edema on the 10th of the admission. MRI of the orbits and paranasal sinuses, revealed soft tissue involvement in retrobulbar regions and paranasal sinusitis. A nasal biopsy confirmed a mucormycosis infection. Another report by Ehrenreich 
[41], described rhino-orbital mucormycosis with orbital compartment syndrome as COVID-19 comorbidity in a 33 years old female, with bilateral maxillary and ethmoid sinus involvement in CT scan.

We hypothesize extensive use of steroids or other immunomodulatory agents, wide spectrum antibiotics administration along with edematous mucosa during upper respiratory tract infection can explain as causes of sinuses and orbital fungal superinfection. Also, untreated DM and diabetic ketoacidosis has been mentioned for susceptibility to both mucormycosis and COVID-19.

\section{Herpes zoster ophthalmicus (HZO)}

$\mathrm{HZO}$ as a result of the varicella zoster virus (VZV) reactivation, commonly involves the ophthalmic division of the trigeminal nerve [42]. In a study by Nofal., et al. [43], 4 patients with COVID-19 infection and a previous episode of varicella, had HZO manifestations. The significant points of this study were the age of the patients as 7, 9, 28 and 42 years old and their immunocompetency, however, the incidence of HZO is high in older patients and weak immune systems [42]. None of these patients had known trigger factors for reactivation of VZV. Complete blood count (CBC) showed lymphopenia in all patients in this report which is a common finding in COVID-19 patients. We hypothesize this lymphopenia along with psychological stress from the disease and the administration of immunomodulatory drugs can put the patient at higher risk for HZO.

\section{Ocular inflammation and uveitis}

Unilateral panuveitis in a 60 years old woman with definitive diagnosis of COVID-19, drew the attention of Benito and colleagues to the linkage between them [32]. Also, as we reviewed the studies on ophthalmic manifestations of COVID-19, encountered the interesting association with white dot syndromes (WDS) spectra [44].

Interferon Beta-1a (IFN-beta1-a), an immunomodulatory agent with antiviral effects, has shown in vivo and in vitro activities on COVID-19 [45]. Drug induced uveitis following the use of these immunomodulators could be a hypothesis for possible association between COVID-19 and ocular inflammatory disorders, however, CoV like any other infection, can act as a trigger to cause or exacerbate uveitis.

\section{Therapeutic-prophylactic measures ocular side effects}

Some ocular symptoms experienced by COVID-19 patients are closely related to drug-induced toxicities. Some drugs that are ap- proved or under evaluation for the treatment of COVID-19 such as antiviral agents, chloroquine/hydroxychloroquine (CQ/HCQ), lopinavir/ritonavir and other HIV protease inhibitors, immunomodulators including corticosteroids, interferons (alpha, beta), etc. can induce ocular side effects [46].

\section{Chloroquine/hydroxychloroquine (CQ/HCQ) ocular toxicity}

In the absence of evidence based proven prophylactic or therapeutic options, CQ/HCQ patented as a considerable choice in COVID-19 treatment or prophylaxis, which raised concerns about drug poisoning especially ocular toxicity. The most common and well-known adverse effect of CQ/HCQ is ocular toxicity, typically retinopathy that may progress to photoreceptor degeneration [47]. Other less important ocular adverse effects including: vortex keratopathy or corneal verticillata (subepithelial deposition of the drug), posterior subcapsular lens opacity and ciliary body dysfunction [48]. According to the study by Bagheri M., et al. ophthalmic consult for prescribed CQ/HCQ as therapeutic-prophylactic option in COVID-19 patients is not recommended except in high-risk patients for retinal toxicity because of the short time prescription and the insignificant cumulative dose of the drug [5].

\section{Antiviral retinal toxicity}

In a study by Roe., et al. three patients with Human immunodeficiency virus (HIV) infection developed a retinal pigment epitheliopathy, macular telangiectasis and intraretinal crystal deposits following administration of ritonavir [49]. However, no report of antiviral ocular toxicity in COVID-19 patients has been published so far, similar side effects are possible when prescribing these drugs to COVID-19 patients. Ocular side effects have also been showed with other drugs like interferons, and interleukin-1(IL-1) and IL-6 inhibitors [16].

Tada A., et al. demonstrated bilateral retinopathy as an ocular adverse effect of IL-6 prescription, which presented with multifocal retinal hemorrhages and cotton-wool spots [50]. Also, bilateral papilledema, HTLV-1 uveitis, viral conjunctivitis, and HZO prescribed as ocular complications in high dose interferon alpha administration [51].

Corticosteroid therapy ocular side effect

In a study by Shah., et al. a 24 years old male developed a central serous chorioretinopathy (CSC) following administration of prednisolone (60 mg/day) for 3 days [52]. Alkin., et al. reported a 
54 years old male with non-arteritic anterior ischemic optic neuropathy received methylprednisolone, $1 \mathrm{gr} /$ day intravenously, for 3 days, and $1 \mathrm{mg} / \mathrm{kg} /$ day orally for a week after that. He developed CSC six days after treatment [53]. Corticosteroids therapy is the most common medical cause of the CSC which is commonly prescribed to COVID-19 patients.

\section{Discussion}

COVID-19 may present ocular symptoms in a variety of different ways. It involves the cornea and conjunctiva and keratoconjunctivitis may consequence. Sometimes, ocular manifestations could result from systemic problems like high positive pressure ventilation and water-electrolyte imbalance. In addition, some COVID-19 medications have ocular side effects [54]. In this paper, we pointed to some COVID-19 unusual ophthalmic presentations which classified into three categories: direct infectious involvement, indirect disease complications, and ocular side effects of prescribed therapeutic-prophylactic options, as mentioned above. To our knowledge, COVID-19 may be coincidental or an underlying factor to the pathogenesis of them. In each part, an attempt was made to explain the mechanism of ocular involvement by reviewing previous studies, or in unknown cases we expressed our hypotheses about them.

Clinicians should have a high index of suspicion for ophthalmic presentations in COVID-19 patients which includes a spectrum of symptomatology from ocular irritation to visual loss.

Immunodeficiency, diabetic ketoacidosis (DKA), broad spectrum antibiotics, and immunomedulatory therapy are the most important predisposing factors for the development of these ocular side effects [55].

\section{Conclusion}

This review represents a panoramic spectra of COVID-19 ophthalmic symptomatology with possible underlying pathophysiology and our hypothesis on mechanism of ocular involvements and accentuates the need to recognize the clinical ophthalmologic presentations associated with this pandemic for more accurate diagnosis.

\section{Conflict of Interest}

The authors of this manuscript declare no conflict of interest in the preparation of this manuscript.

\section{Bibliography}

1. "Organisation WH. Statement on the second meeting of the international health regulations (2005) emergency committee regarding the outbreak of novel coronavirus (2019-nCoV) (2020).

2. Adhikari SP., et al. "Epidemiology, causes, clinical manifestation and diagnosis, prevention and control of coronavirus disease (COVID-19) during the early outbreak period: a scoping review". Infectious Diseases of Poverty 9 (2020).

3. Li J., et al. "Novel Coronavirus disease 2019 (COVID-19): The importance of recognising possible early ocular manifestation and using protective eyewear". ed: BMJ Publishing Group Ltd (2020).

4. Bagheri M., et al. "A Review on 2019 Novel Coronavirus Pneumonia in Ophthalmology". Ocular Immunology and Inflammation 28 (2020): 909-915.

5. Bagheri M., et al. "Is ocular toxicity expected in chloroquine/ hydroxychloroquine prescription as a therapeutic or prophylactic option in COVID-19?". Recent Patents on Anti-Infective Drug Discovery 15 (2020): 113-118.

6. Xie X., et al. "How far droplets can move in indoor environments-revisiting the Wells evaporation-falling curve". Indoor Air 17 (2007): 211-225.

7. Wu P., et al. "Characteristics of ocular findings of patients with coronavirus disease 2019 (COVID-19) in Hubei Province, China". JAMA Ophthalmology 138 (2020): 575-578.

8. Hong N., et al. "Evaluation of ocular symptoms and tropism of SARS-CoV-2 in patients confirmed with COVID-19". Acta Ophthalmologica 98 (2020): e649-55.

9. Loon SC., et al. "The severe acute respiratory syndrome coronavirus in tears". British Journal of Ophthalmology 88 (2004): 861-863.

10. Ozturker ZK. "Conjunctivitis as sole symptom of COVID-19: A case report and review of literature". European Journal of Ophthalmology (2020).

11. Turbin RE., et al. "Orbital cellulitis, sinusitis and intracranial abnormalities in two adolescents with COVID-19". Orbit (2020): 1-6. 
12. Guan WJ., et al. "Clinical characteristics of coronavirus disease 2019 in China”. New England Journal of Medicine 382 (2020): 1708-1720.

13. Ho D., et al. "COVID-19 and the Ocular Surface: A Review of Transmission and Manifestations". Ocular Immunology and Inflammation 28 (2020): 726-734.

14. Xia J., et al. "Evaluation of coronavirus in tears and conjunctival secretions of patients with SARS-CoV-2 infection". Journal of Medical Virology 92 (2020): 589-594.

15. Ping W., et al. "Characteristics of ocular findings of patients with coronavirus disease 2019 (COVID-19) in Hubei Province, China". JAMA Ophthalmology 138 (2020): 575.

16. Bertoli F., et al. "Ocular Findings in COVID-19 Patients: A Review of Direct Manifestations and Indirect Effects on the Eye". Journal of Ophthalmology (2020).

17. Cheema M., et al. "Keratoconjunctivitis as the initial medical presentation of the novel coronavirus disease 2019 (COVID-19)". The Canadian Journal of Ophthalmology 55 (2020): 125-129.

18. Guo D., et al. "Relapsing viral keratoconjunctivitis in COVID-19: a case report". Virology Journal 17 (2020): 97.

19. Dumitrascu OM., et al. "Acute ophthalmic artery occlusion in a COVID-19 patient on apixaban". The Journal of Stroke and Cerebrovascular Diseases 29 (2020):104982.

20. Gaba WH., et al. "Bilateral Central Retinal Vein Occlusion in a 40-Year-Old Man with Severe Coronavirus Disease 2019 (COVID-19) Pneumonia". American Journal of Case Reports 21 (2020): e927691.

21. Walinjkar JA., et al. "Central retinal vein occlusion with COVID-19 infection as the presumptive etiology". Indian Journal of Ophthalmology 68 (2020): 2572-2574.

22. Selvaraj V., et al. "Acute Vision Loss in a Patient with COVID-19". Rhode Island Medical Journal 103 (2013): 37-38.

23. Shah A., et al. "OCT angiography features of paracentral acute middle maculopathy". Indian Journal of Ophthalmology 67 (2019): 417-419.
24. Gascon P., et al. "Covid-19-Associated Retinopathy: A Case Report". Ocular Immunology and Inflammation 16 (2020): 12931297.

25. Tang N., et al. "Abnormal coagulation parameters are associated with poor prognosis in patients with novel coronavirus pneumonia". Journal of Thrombosis and Haemostasis 18 (2020): 844-847.

26. Llitjos JF., et al. "High incidence of venous thromboembolic events in anticoagulated severe COVID-19 patients". Journal of Thrombosis and Haemostasis (2020).

27. Bikdeli B., et al. "COVID-19 and Thrombotic or Thromboembolic Disease: Implications for Prevention, Antithrombotic Therapy, and Follow-Up: JACC State-of-the-Art Review". Journal of the American College of Cardiology 75(2020): 2950-2973.

28. Cyr DG., et al. "Severe Bilateral Vision Loss in 2 Patients With Coronavirus Disease 2019". Journal of Neuro-Ophthalmology 40 (2020): 403-405.

29. Belghmaidi S., et al. "Third Cranial Nerve Palsy Presenting with Unilateral Diplopia and Strabismus in a 24-Year-Old Woman with COVID-19". American Journal of Case Reports 21 (2020): e925897.

30. Dinkin M., et al. "COVID-19 presenting with ophthalmoparesis from cranial nerve palsy”. Neurology 95 (2020): 221-223.

31. Greer CE., et al. "Isolated Cranial Nerve 6 Palsy in 6 Patients With COVID-19 Infection". Journal of Neuro-Ophthalmology 40 (2020): 520-522.

32. Benito-Pascual B., et al. "Panuveitis and Optic Neuritis as a Possible Initial Presentation of the Novel Coronavirus Disease 2019 (COVID-19)". Ocular Immunology and Inflammation 28 (2020): 922-925.

33. Stevens DV., et al. "Complications of Orbital Emphysema in a COVID-19 Patient”. Ophthalmology 127 (2020): 990.

34. Shah P., et al. "Non-traumatic Orbital Emphysema following suspected COVID-19 infection". Journal of Clinical Ophthalmology 4 (2020): 294-296.

35. Das D., et al. "Unilateral orbital emphysema in a COVID-19 patient". Indian Journal of Ophthalmology 68 (2020): 2535. 
36. McGuire H., et al. "Extraconal orbital emphysema secondary to barotrauma in a ventilated patient with COVID-19". Anaesthesia Reports 8 (2020): 111-112.

37. Bhat M., et al. "Think Twice Before You Blow-Case Report And Review Of Literature". Journal of Clinical and Diagnostic Research 4 (2010): 3558-3560.

38. McGuire HD., et al. "Extraconal orbital emphysema secondary to barotrauma in a ventilated patient with COVID-19". Anaesthesia Reports 8 (2020): 111-112.

39. Koparal M., et al. "Assessment of mucociliary clearance as an indicator of nasal function in patients with COVID-19: a crosssectional study". European Archives of Oto-Rhino-Laryngology (2020): 1-6.

40. Mehta S., et al. "Rhino-orbital mucormycosis associated with COVID-19". Cureus 12 (2020).

41. Werthman-Ehrenreich A., "Mucormycosis with orbital compartment syndrome in a patient with COVID-19". The American Journal of Emergency Medicine (2020).

42. Borkar DS., et al. "Incidence of herpes zoster ophthalmicus: results from the Pacific Ocular Inflammation Study". Ophthalmology 120 (2013): 451-456.

43. Nofal A., et al. "Herpes zoster ophthalmicus in COVID-19 patients". International Journal of Dermatology 59 (2020): 15451546.

44. Ortiz-Seller A., et al. "Ophthalmic and Neuro-ophthalmic Manifestations of Coronavirus Disease 2019 (COVID-19)". Ocular Immunology and Inflammation 28 (2020): 1285-1289.

45. Baghaei P., et al. "Combination therapy of IFN $\beta 1$ with lopinavirritonavir, increases oxygenation, survival and discharging of sever COVID-19 infected inpatients". International Immunopharmacology 92 (2020): 107329.

46. “2021, COVID-19 Treatment Guidelines Panel. Coronavirus Disease 2019 (COVID-19) Treatment Guidelines (2021).

47. Rosenthal A., et al. "Chloroquine retinopathy in the rhesus monkey". Investigative Ophthalmology and Visual Science 17 (1978): 1158-1175.
48. Stokkermans T., et al., "Chloroquine and hydroxychloroquine toxicity.[Updated 2019 Jun 4]”. StatPearls. Treasure Island (FL): StatPearls Publishing (2020).

49. Roe RH., et al. "Retinal pigment epitheliopathy, macular telangiectasis, and intraretinal crystal deposits in HIV-positive patients receiving ritonavir". Retina 31 (2011): 559-565.

50. Tada A., et al. "Anti-interleukin-6 receptor antibody therapy-induced retinopathy in a patient with rheumatoid arthritis". Case Reports in Rheumatology 2012 (2012): 270315.

51. Kadayifcilar S., et al. "Ocular complications with high-dose interferon alpha in chronic active hepatitis". Eye 13(1999): 241246.

52. Shah SP., et al. "Steroid-induced central serous retinopathy". Indian Journal of Pharmacology 43 (2011): 607-608.

53. Alkin Z., et al. "Steroid-induced central serous chorioretinopathy in a patient with non-arteritic anterior ischemic optic neuropathy". Saudi Journal of Ophthalmology 29 (2015): 232-234.

54. Wong RLM., et al. "COVID-19: Ocular Manifestations and the APAO Prevention Guidelines for Ophthalmic Practices". The Asia-Pacific Journal of Ophthalmology (2020).

55. Binder U., et al. "Mucormycosis - from the pathogens to the disease". Clinical Microbiology and Infection 20 (2014): 60-66.

\section{Assets from publication with us}

- Prompt Acknowledgement after receiving the article

- Thorough Double blinded peer review

- Rapid Publication

- Issue of Publication Certificate

- High visibility of your Published work

Website: www.actascientific.com/

Submit Article: www.actascientific.com/submission.php Email us: editor@actascientific.com

Contact us: +919182824667 